\title{
From economic crisis to the COVID-19 pandemic crisis: evidence from a mental health helpline in Greece
}

\author{
Lily Evangelia Peppou ${ }^{1,2} \cdot$ Marina Economou $^{1,2} \circledast$. Theodora Skali ${ }^{1} \cdot$ Charalampos Papageorgiou ${ }^{1}$
}

Received: 22 April 2020 / Accepted: 7 July 2020 / Published online: 14 July 2020

c) Springer-Verlag GmbH Germany, part of Springer Nature 2020

Keywords COVID-19 $\cdot$ Mental health help line $\cdot$ Pandemic crisis · Greece

Past experiences on viral epidemics have underscored the importance of considering mental health an integral part of any health response to biological disasters, including the COVID-19 outbreak [1]. In this context, telephone helplines are deemed crucial for timely and effective treatment of emerging mental health issues as well as emergency cases [2]. Despite its significance, there is dearth of research on the mental health effects of the COVID-19 pandemic, while there are no data at all emanating from telephone helplines.

According to data from the European Centre for Disease Prevention and Control, until 21st of April, 1.073.947 COVID-19 cases have been identified in Europe and 103.989 deaths [3]. In Greece, figures have remained as low as 2.401 cases with 121 deaths, largely due to the timely public health measures to prevent the spreading of the virus [4]. In this context, the establishment of a nationwide Telephone Helpline for the Psychosocial Support amid COVID-19, operated by the First Department of Psychiatry, University of Athens, has been an immediate response to the pandemic in Greece. Here, we present data from a random sample of 576 out of 1.728 calls we received during a 3 -week period since the outset of the restrictive measures. The Helpline operates on a daily basis, including weekends, from 10:00 to 22:00, and it consists of psychologists and psychiatrists. Concomitantly, upon completion of the call, the following information was garnered for service evaluation and research purposes: callers' socio-demographic characteristics (gender,

Marina Economou

marinaeconomou1@gmail.com

1 First Department of Psychiatry, Medical School, Aiginition Hospital, National and Kapodistrian University of Athens, Athens, Greece

2 University Mental Health, Neurosciences and Precision Medicine Research Institute "Costas Stefanis” (UMHRI), Athens, Greece age, place of residence, family status, living arrangement, education, and employment status), reason for calling, beliefs about the coronavirus, mental health status, media exposure, frequency of experienced emotions, and mode of intervention provided. The research protocol had been approved by the Ethics Committee of the Frist Department of Psychiatry, University of Athens-Eginition Hospital. This letter presents preliminary data regarding the content of calls and mental health status.

Among the 576 calls, only 7 (1.2\%) callers were confirmed cases of COVID-19. Concerning their content, most calls pertained to the quarantine (482 calls, $83.7 \%$ ), and more specifically to feelings of restraint (56\%) and loneliness (52.9\%). Moreover, 463 calls (80.4\%) were centred on COVID-19 per se. Among them, the most frequent complaint was fear of illness, with $37.4 \%$ of calls being made by people not vulnerable to COVID-19 and $26.6 \%$ by people who were. It merits noting that for $19.4 \%$ of COVID-19 pertinent calls, primary concern was directed at the health of beloved ones. Finally, 175 calls (30.4\%) were germane to anxiety over the economy. In one out of three calls (36.5\%), the belief that the virus is unpredictable was ubiquitous.

As regards emerging mental health issues, Goldberg screening tools for anxiety and depression were employed [5] consonant with research on helpline counseling services both in Greece [6] and worldwide [7]. Additionally, previous use of the scales in the Depression Helpline in Greece amid the recession was the main reason for opting for these screening tools, as comparisons between these two time periods are enabled. It merits noting that at the time which the helpline started its operation, there was no COVID-19 related instrument available in the country. Evidence on symptom prevalence during the previous 2 weeks (Table 1) corroborates the superiority of anxiety symptoms over depressive ones; however, a greater percentage of callers is above the threshold for clinically important depression than 
Table 1 Prevalence of anxiety and depressive symptoms

\begin{tabular}{|c|c|c|c|}
\hline & Number of calls (\%) & Mean (st.dev) & $\begin{array}{l}\text { Clinically } \\
\text { significant } \\
\text { cases }\end{array}$ \\
\hline \multicolumn{4}{|l|}{ Anxiety scale } \\
\hline Keyed up, on edge & $160(27.8 \%)$ & & \\
\hline Worrying a lot & $283(49.1 \%)$ & & \\
\hline Irritability & $126(21.9 \%)$ & & \\
\hline Difficulty in relaxing & $311(54 \%)$ & & \\
\hline Poor sleep & $142(24.7 \%)$ & & \\
\hline Headaches, neck aches & $44(9.2 \%)$ & & \\
\hline Autonomic symptoms & $102(17.7 \%)$ & & \\
\hline Health worries & $250(43.4 \%)$ & & \\
\hline Delayed sleep & $118(20.5 \%)$ & & \\
\hline Anxiety scale score & & $3.19(2)$ & $117(20.3 \%)$ \\
\hline \multicolumn{4}{|l|}{ Depression scale } \\
\hline Low energy & $139(24.1 \%)$ & & \\
\hline Loss of interest & $139(24.1 \%)$ & & \\
\hline Loss of confidence & $119(20.7 \%)$ & & \\
\hline Hopelessness & $176(30.6 \%)$ & & \\
\hline Inefficient thinking & $150(26 \%)$ & & \\
\hline Poor appetite/weight loss & $22(3.8 \%)$ & & \\
\hline \multicolumn{4}{|l|}{ Early waking } \\
\hline Felt slowed up & $63(10.9 \%)$ & & \\
\hline Felt worse in the morning & $41(7.1 \%)$ & & \\
\hline Depression scale & $56(15.8 \%)$ & $2.55(1.7)$ & $213(37 \%)$ \\
\hline Depression and anxiety & & & $52(9.2 \%)$ \\
\hline Panic attack & & & $134(23.3 \%)$ \\
\hline
\end{tabular}

for clinically important anxiety (37\% vs. 20.3\%), triggering thus contemplation about the nature of anxiety. Are the anxiety symptoms reflecting trait anxiety, subthreshold anxiety disorders, or precursors of generalized anxiety disorder? One out of ten callers $(9.2 \%)$ scored above the threshold for both dimensions. Moreover, $23.3 \%$ of the callers experienced a panic attack during the previous 2 weeks. For the majority of callers $(359,62.3 \%)$, active and empathetic listening, emotional support, and the delivery of psychoeducation (provision of evidence-based information and problem solving skills training) were the main course of action on the part of the professionals. For 217 callers (37.7\%), a follow-up call was recommended, while a slim minority 35 (6.1\%) was referred to emergency mental health units.

In Greece, the COVID-19 pandemic has been largely contained, rendering the country exemplary. This is also reflected in the small representation of COVID-19 cases in our sample. This is, perhaps, the reason why more calls address the restrictive measures than the COVID-19 per se, while fears for the economy are reminiscent of the prior financial crisis. While at first glance, anxiety disorders should be the focal point for interventions, the insidious course of depression should not be overlooked, especially in light of the heightened prevalence rates of the disorder amid the recession in the country [8]. It is noteworthy that 1 -month prevalence of major depression increased from $3.3 \%$ in 2008 to $12.3 \%$ in 2013 as result of the enduring financial crisis. Therefore, it remains to be seen whether the current COVID-19 crisis will re-activate the previous cases of depression or it will generate new ones. Will the new cases be accounted for by the restrictive measures or will anxiety inevitably turn into depression, congruent with contemporary neurocognitive evidence [9] corroborating the development of depression in individuals high in trait anxiety, when faced with adversity? At the same time, for 1 out of 10 callers, clinical anxiety and clinical depression appear to co-exist, lending further support to the intricate interrelations between the two clinical entities [10]. Despite the lingering controversy over the two conditions, their cooccurrence embedded in a biopsychosocial model lends further credence to the subjective suffering amid the COVID19 outbreak. Further data from the Helpline coupled with evidence from general population surveys will shed light on the underpinnings of these findings.

Taken together, these findings illuminate the ways, whereby people cope with adversity and uncertainty. The COVID-19 pandemic has triggered worry and stress, in the form of anxiety symptoms. In some people, these are 
organized into a clinical syndrome. In some others, it remains to be seen whether anxiety gradually gets transformed into major depression or depression follows a distinct pathway. These emerging mental health problems necessitate the timely delivery of effective interventions. Mental health helplines have a wider coverage than online services due to reaching people in areas where access to Internet may be hampered. At the same time, they play an important role in screening for common mental disorders, alleviating anxiety symptoms among those without mental health conditions and networking with other services, like online platforms, for the delivery of brief psychotherapy or for referring emergency cases. Psychoeducational techniques jointly with emotional support and empathetic listening may contribute in exacerbating stress and mental health symptoms among callers. Nonetheless, mental health helplines should be embedded in a multipronged network of telepsychiatry services. On top of that, a multifaceted approach, including health education interventions and initiatives to counteract fake news as well as, incentivizing people to keep healthy habits, implement routines, being socially connected and exert a degree of control over their micro-environment is imperative for successfully mitigating the mental health effects of the COVID-19 pandemic.

Authors' contributions LP: conception, design, and interpretation of data; drafting the letter. ME: conception, design, and interpretation of data; critically revising the letter. TS: data acquisition; critically revising the letter. CP: conception, design, and critically revising the letter.

Funding Both the Helpline and the study were funded by the Regional Governor of Attica.

\section{Compliance with ethical standards}

Conflict of interest The authors declare no conflict of interest.
Ethics committee approval The study has received ethical approval by the Ethics Committee of the First Department of Psychiatry, Aiginition Hospital, National and Kapodistrian University of Athens, and it was conducted in accordance with the ethical standards delineated in the Helsinki Declaration.

\section{References}

1. Xiang Y-T, Li W, Zhang Q et al (2020) Timely research papers about COVID-19 in China. Lancet 395:684-685

2. Liu N, Zhang F, Wei C, et al (2020) Prevalence and predictors of PTSS during COVID-19 Outbreak in China hardest-hit areas: gender differences matter. Psychiatry Res 287:112921-112927

3. European Centre for Disease Prevention and Control (2020) Situation update worldwide. https://www.ecdc.europa.eu/en/geogr aphical-distribution-2019-ncov-cases. Accessed 21 Apr 2020

4. Hellenic Government (2020) Daily Covid-19 Update in Greece. https://covid19.gov.gr/covid19-live-analytics/. Accessed $21 \mathrm{Apr}$ 2020

5. Goldberg D, Bridges K, Duncan-Jones P, Grayson D (1988) Detecting anxiety and depression in general medical settings. BMJ 297:897-899

6. Economou M, Peppou LE, Louki E, et al (2012) Depression Telephone Helpline: Help seeking during the financial crisis. Psychiatriki 23:17-28 [in Greek]

7. Burgess N, Christensen H, Leach LS et al (2008) Mental health profile of callers to a telephone counselling service. J Telemed Telecare 14:42-47

8. Economou M, Angelopoulos E, Peppou LE et al (2016) Enduring financial crisis in Greece: prevalence and correlates of major depression and suicidality. Soc Psychiatry Psychiatr Epidemiol 51:1015-1024

9. Sandi C, Richter-Levin G (2009) From high anxiety trait to depression: a neurocognitive hypothesis. Trends Neurosci 32:312-320

10. Gorman JM (1996) Comorbid depression and anxiety spectrum disorders. Depress Anxiety 4:160-168 